
\title{
Reseacch S Suare \\ New Diagnostic Parameter for Neonatal Sepsis: Red Cell Distribution Width Fluctuations
}

\author{
Bin-Fang Guo ( $\nabla$ gbf1112@126.com ) \\ Cangzhou Central Hospital https://orcid.org/0000-0001-8217-7333 \\ Yu-Chun Wang \\ Cangzhou Central Hospital \\ Ping-Ping $X u$ \\ Cangzhou Central Hospital
}

Research

Keywords: red blood cell distribution width, sepsis, neonate, diagnosis

Posted Date: August 12th, 2021

DOI: https://doi.org/10.21203/rs.3.rs-789540/v1

License: (1) This work is licensed under a Creative Commons Attribution 4.0 International License.

Read Full License 


\section{Abstract}

Background: To evaluate the role of red cell distribution width (RDW) fluctuations in the diagnosis of neonatal sepsis.

Methods: In this retrospective cohort study of the Medical Information Mart for Intensive Care III database (version 1.4), newborns with sepsis were matched with patients without sepsis to analyze RDW as a diagnostic marker. Those newborns were analyzed based on gestational age, sex, and ethnicity. Receiver operating characteristic (ROC) analysis and multiple logistic regression were used to evaluate RDW fluctuation for sepsis diagnosis.

Results: A total of 2456 neonates were included. RDW fluctuations showed better accuracy than the maximum RDW did (area under the curve (AUC): 0.88 vs. 0.81 , sensitivity $92.10 \%$ vs. $78.70 \%$; specificity $71.70 \%$ vs. $69.30 \%$, respectively). Furthermore, logistic regression analysis showed a positive association between RDW fluctuation and the diagnosis of sepsis (odds ratio 1.13; 95\% confidence interval, 1.081.18) after adjusting for gestational age, hemoglobin, and white blood cell count.

Conclusion: RDW fluctuations might be a potential diagnostic marker for NICU patients with sepsis.

\section{Introduction}

Neonatal sepsis is a common and lethal systemic disease occurring in the first four weeks of life. Early recognition and treatment of sepsis can prevent Systemic Inflammatory Response Syndrome and its associated morbidity and mortality [1]. However, the clinical manifestations of neonatal septicemia are nonspecific, which decrease the rate of early diagnosis. Clinicians identify septicemia mainly based on positive blood culture results. However, blood culture tests have some limitations, such as high false negative rates associated with maternal antibiotic use, low microbial loads (low extracted blood volume especially from preterm infants), high false-positive results from contamination, long time to obtain results $(48-72 \mathrm{~h})$, and low sensitivity rate $(19.2 \%)[1]$.

Red cell distribution width (RDW) is a hematologic index reflecting red blood cell (RBC) volume heterogenicity [2]. It is a useful index in many hematologic diseases [3] and can vary in several inflammatory diseases. Adult studies indicated that RDW can reflect the inflammatory condition, just like C-reactive protein (CRP) and erythrocyte sedimentation rate [4, 5]. In newborns, a strong positive correlation was found between RDW and CRP $(r=0.8 ; P<0.0001)$ [6]. However, single RDW had a low specificity for diagnosing sepsis, whereas the comprehensive analyses of RDW, platelet distribution width, and neutrophil-lymphocyte count ratio exhibited a higher diagnostic accuracy (an area under the curve, 0.704) [7].

A large number of studies has investigated the prognostic value of RDW [8]. Of note, it is rarely used in sepsis neonatal diagnostic models. To date, the significance of fluctuating RDW in the diagnosis of 
neonatal sepsis is still unclear. Thus, in this study, we evaluated the applicability of fluctuating RDW values in the early diagnosis of neonatal sepsis.

\section{Materials And Methods}

\section{Database and Patients}

This is a retrospective study collected from an open-access large critical care database, Medical Information Mart for Intensive Care III (MIMIC-III) database (version 1.4). MIMIC III comprised 38645 adults and 7875 neonates admitted to the intensive care unit (ICU) of Beth Israel Deaconess Medical Center between 2001 and 2012 [9]. This database was approved by the Institutional Review Board (IRB) at the Massachusetts Institute of Technology. One of the authors (Bin-Fang Guo, certification number: 36077987) passed the National Institute of Health web-based training course and the Protecting Human Research Participants Examination and was approved to extract data from MIMIC III for research purposes. Informed consent was waived because all data are from publicly available resources.

The inclusion criteria of this study were as follows: (1) newborns admitted in the neonatal ICU (NICU) within 24 hours after birth with gestational age $<42$ weeks; (2) recorded RDW results. If a patient had multiple hospitalizations, the first hospitalization was included in the analysis. The exclusion criteria were as follows: (1) missing RDW data; (2) presence of congenital malformation and neonatal asphyxia. Patients with sepsis (sepsis group) were identified from those without sepsis (control group).

In cases with more than one RDW value in the NICU, RDW fluctuation (\%) was calculated as follows: (maximum RDW - initial RDW) / initial RDW.

\section{Data Extraction}

Clinical and demographic data were stored in 26 tables extracted using structured query language, which include age at admission, sex, gestational age, birth weight, delivery mode, hematologic parameters, diagnosis, and in hospital mortality. The initial complete blood count, maximum RDW value, and initial RDW values were also obtained. We used the keywords "bacteremia of newborn" and "septicemia [sepsis] of newborn" to search patients with sepsis in MIMIC III.

Some variables had $<5 \%$ missing values. The regression padding method was used to impute missing values in birth weight. Median was used to impute missing values in white blood cell (WBC) and length of stay in NICU (LOS) using Stata.

\section{Statistical Analysis}

Quantitative variables in each group are expressed as median (first quartile, third quartile), and differences between groups were identified using the Mann-Whitney $U$ test for non-normal distribution. Categorical variables were expressed as number and percentage, and comparisons between groups were made using the chi-square test or Fisher's exact test as appropriate. 
The infants in the control group were further divided into five groups according to their gestational age (GA) in weeks s (group A: GA < 28; group B: $32>G A \geq 28$; group C: $34>G A \geq 32$; group D: $37>G A \geq 34$; group $E: G A \geq 37$ ). The effect of GA on RDW was assessed using the Kruskal-Wallis for multiple groups. The effect of ethnicity on RDW was also assessed.

A nested 1:1 matching case-control study was used to minimize the selection bias between the sepsis and control groups, excluding patients with only one RDW result. Patients were matched in a 1:1 ratio based on GA, sex, and ethnicity. Data were compared between groups using the paired Wilcoxon ranksum test and paired chi-square test as appropriate.

The receiver operating characteristic curve (ROC) was used to evaluate the diagnostic value of RDW for sepsis. Univariate and multivariate logistic regression were used to determine the significant predictors of RDW. We developed multivariable logistic regression models for the case-control matching groups. Variables with $P<0.2$ in the univariate analysis were further incorporated into multivariate logistic regression models. The odds ratios and $95 \%$ confidence intervals $(95 \% \mathrm{Cls})$ were calculated.

Statistical analysis was performed using IBM SPSS version 26.0 (IBM, Armonk, NY, USA)and GraphPad Prism8 (GraphPad Software Inc., CA). A $P$ value $<0.05$ was considered statistically significant.

\section{Results}

\section{Baseline characteristics}

Of the 7875 newborns that were extracted from the MIMIC- III database, the following patients were excluded: 600 with congenital disease, 17 with neonatal asphyxia, 1051 with ICU stays less than $24 \mathrm{~h}, 14$ with incomplete NICU records, 2040 without major laboratory results, 2567 with unknown or inaccurate GA, 24 neonates with GA $>42$ weeks, and 157 neonates delivered $>24 \mathrm{~h}$. Finally, 2456 eligible patients were enrolled (Fig. 1). The clinical characteristics of the patients are summarized in Table 1. We classified 168 patients into the sepsis group and 2288 patients into the control group. 
Table 1

Characteristics of groups before matching

\begin{tabular}{|c|c|c|c|}
\hline \multirow[t]{2}{*}{ Contents } & Sepsis group & Control group & $P$ \\
\hline & $N=168$ & $N=2288$ & \\
\hline GA, week (IQR) & $33.5(30.00,34.50)$ & $34.50(33.50,34.50)$ & $<0.01$ \\
\hline BW, g (IQR) & $1.03(0.73,1.43)$ & $2.13(1.59,2.13)$ & $<0.01$ \\
\hline Age, h (IQR) & $14.00(7.00,19.00)$ & $13.00(8.00,19.00)$ & 0.72 \\
\hline Male, n (\%) & $92(54.80)$ & $1272(55.60)$ & 0.83 \\
\hline Caesarean section, n (\%) & $115(68.50)$ & $1557(68.10)$ & 0.91 \\
\hline Ethnicity, n (\%) & & & 0.11 \\
\hline Caucasians & $95(56.50)$ & $1419(62.00)$ & \\
\hline Asian & $7(4.20)$ & $153(6.70)$ & \\
\hline Black & $30(17.90)$ & $265(11.60)$ & \\
\hline Hispanic/Latino & $10(6.00)$ & $121(5.30)$ & \\
\hline Unknown/other & $26(15.50)$ & $330(14.40)$ & \\
\hline \multicolumn{4}{|l|}{ Haematology } \\
\hline WBC, $10^{9} / \mathrm{L}(\mathrm{IQR})$ & $8.05(5.40,13.20)$ & $11.60(8.60,15.80)$ & $<0.01$ \\
\hline Neutrophil, \%(IQR) & $28.00(16.00,42.00)$ & $36.00(24.00,53.00)$ & $<0.01$ \\
\hline Lymphocyte, \%(IQR) & $55.05(40.00,71.00)$ & $50.00(33.00,64.00)$ & $<0.01$ \\
\hline NLR(IQR) & $0.50(0.23,1.00)$ & $0.72(0.38,1.60)$ & $<0.01$ \\
\hline Haemoglobin, g/L(IQR) & $14.85(13.30,16.60)$ & $16.30(14.90,17.70)$ & $<0.01$ \\
\hline RDW_initial, \%(IQR) & $16.80(16.10,17.75)$ & $17.00(16.40,17.80)$ & 0.06 \\
\hline RDW_max, \%(IQR) & $19.30(17.65,21.70)$ & $17.20(16.50,18.10)$ & $<0.01$ \\
\hline RDW_min, \%(IQR) & $16.40(15.70,17.20)$ & $16.90(16.30,17.70)$ & $<0.01$ \\
\hline $\mathrm{PLT}, 10^{9} / \mathrm{L}(\mathrm{IQR})$ & $230.00(184.00,299.00)$ & $266(220.00,318.00)$ & $<0.01$ \\
\hline
\end{tabular}

GA: gestation age; BW: birth weight; WBC: White Blood Cells; NLR: Neutrophil to Lymphocyte ratio; RDW_initial: The first Red Cell Distribution Width after admission to NICU; RDW_max: The maximum of RDW detected in NICU; RDW_min: The minimal of RDW detected in NICU; PLT: Platelet; RPR:

RDW_initial to platelet ratio; RDS: Respiratory Distress Syndrome PDA: Patent Ductus Arteriosus; LOS: Length of stay in NICU 


\begin{tabular}{|c|c|c|c|}
\hline \multirow[t]{2}{*}{ Contents } & Sepsis group & Control group & \multirow[t]{2}{*}{$P$} \\
\hline & $N=168$ & $N=2288$ & \\
\hline $\mathrm{RPR}(\mathrm{IQR})$ & $0.07(0.06,0.09)$ & $0.06(0.05,0.08)$ & $<0.01$ \\
\hline \multicolumn{4}{|l|}{ Comorbidity } \\
\hline RDS, n (\%) & 136(81.00) & $787(34.40)$ & $<0.01$ \\
\hline PDA, n (\%) & $68(40.50)$ & $186(8.10)$ & $<0.01$ \\
\hline LOS, d(IQR) & $52.63(24.36,87.58)$ & 10.64(3.77,25.05) & $<0.01$ \\
\hline In-hospital mortality, n (\%) & $8(4.80)$ & $24(1.00)$ & $<0.01$ \\
\hline \multicolumn{4}{|c|}{$\begin{array}{l}\text { GA: gestation age; BW: birth weight; WBC: White Blood Cells; NLR: Neutrophil to Lymphocyte ratio; } \\
\text { RDW_initial: The first Red Cell Distribution Width after admission to NICU; RDW_max: The maximum } \\
\text { of RDW detected in NICU; RDW_min: The minimal of RDW detected in NICU; PLT: Platelet; RPR: } \\
\text { RDW_initial to platelet ratio; RDS: Respiratory Distress Syndrome PDA: Patent Ductus Arteriosus; LOS: } \\
\text { Length of stay in NICU }\end{array}$} \\
\hline
\end{tabular}

Patients in the sepsis group had lower median GA and birth weight compared to those in the control group. The sepsis group had significantly higher levels of initial RDW to platelet ratio (RPR), lymphocyte percentage, and maximal RDW, but lower levels of WBC, hemoglobin, platelet, and neutrophil percentage $(P<0.01)$. Initial RDW was not significantly higher in the sepsis group. The sepsis group had longer hospital stay than the control group. The sepsis group had higher rates of respiratory distress syndrome (RDS), Patent Ductus Arteriosus (PDA), or in-hospital mortality than the control group.

The infants had different initial RDW values across the GAs (Fig. 1A). The median of the initial RDW in group A was significantly lower than groups B, C, and D. The median of the initial RDW in newborns with GA between 28 and 37 weeks remained stable, while it decreased with GA of 37 weeks or more.

The majority of the newborns in the control group with GA between 34 and 37 weeks were Caucasians (63.50\%), followed by Asians (7.06\%), Blacks (10.74\%), Hispanic (4.70\%), and unknown in 137 patients (14.01\%). The order of the initial RDW by race was Hispanic > Black > Asian > Caucasians (Fig. 1B).

Except for patients with only one RDW test result, 625 patients were selected for 1:1 matched-pairs casecontrol matching; 254 patients formed 127 pairs for subsequent comparative analyses between the sepsis and control groups (Table 2). The median interquartile range (IQR) birth weight of the sepsis group was significantly higher $0.92 \mathrm{~kg}(0.72,1.24)$ than the control group $1.64 \mathrm{~kg}(1.27,2.43 ; P<0.01)$. The sepsis group had lower median IQR for WBC $[7.30 \times 10 \nabla / L(5.20,11.90)$ vs. $9.50 \times 10 \nabla / L(6.10,13.65)]$, hemoglobin $[14.80 \mathrm{~g} / \mathrm{dL}(13.40,16.40)$ vs. $16.20 \mathrm{~g} / \mathrm{dL}(14.30,17.30)]$, and platelet count $[217.00 \times 10 \mathrm{~V} / \mathrm{L}$ $(177.00,275.00)$ vs. $252.00 \times 10 \otimes / L(193.00,296.50)]$ than the control group (Table 2). 
Table 2

Clinical parameters of the groups after case-control matching

\begin{tabular}{|c|c|c|c|}
\hline & Sepsis group & Control group & $\mathbf{P}$ \\
\hline & $N=127$ & $N=127$ & \\
\hline GA, week (IQR) & $33.50(30.00,34.50)$ & $33.50(30.00,34.50)$ & 0.52 \\
\hline BW, g (IQR) & $0.92(0.72,1.24)$ & $1.64(1.27,2.43)$ & $<0.01$ \\
\hline Age, h (IQR) & $15.00(8.00,19.00)$ & $12.00(5.00,18.00)$ & 0.11 \\
\hline Male, n (\%) & $72(56.70)$ & $72(56.70)$ & 1.00 \\
\hline Caesarean section, n (\%) & $89(70.10)$ & $96(75.60)$ & 0.38 \\
\hline Ethnicity, n (\%) & & & 1.00 \\
\hline Caucasians & $71(55.90)$ & $71(55.90)$ & \\
\hline Asian & $4(3.10)$ & $4(3.10)$ & \\
\hline Black & $25(19.70)$ & $25(19.70)$ & \\
\hline Hispanic/Latino & $8(6.30)$ & $8(6.30)$ & \\
\hline Unknown/other & $19(15.00)$ & $19(15.00)$ & \\
\hline Haematology & & & \\
\hline WBC, $10^{9} / L(I Q R)$ & $7.30(5.20,11.90)$ & $9.50(6.10,13.65)$ & 0.01 \\
\hline Neutrophil, \%(IQR) & $27.00(14.50,40.50)$ & $29.00(18.00,45.50)$ & 0.10 \\
\hline Lymphocyte, \%(IQR) & $56.00(41.50,74.00)$ & $54.00(38.50,71.00)$ & 0.59 \\
\hline $\mathrm{NLR}(\mathrm{IQR})$ & $0.49(0.21,1.00)$ & $0.53(0.26,1.15)$ & 0.39 \\
\hline Haemoglobin, g/L(IQR) & $14.80(13.40,16.40)$ & $16.20(14.30,17.30)$ & $<0.01$ \\
\hline RDW_initial, \%(IQR) & $16.70(16.10,17.70)$ & $17.00(16.20,17.70)$ & 0.67 \\
\hline RDW_max, \%(IQR) & $20.20(18.30,22.00)$ & $17.40(16.50,18.75)$ & $<0.01$ \\
\hline RDW_min, \%(IQR) & $16.40(15.70,17.20)$ & $16.70(15.95,17.50)$ & 0.16 \\
\hline $\mathrm{PLT}, 10^{9} / \mathrm{L}(\mathrm{IQR})$ & $217.00(177.00,275.00)$ & $252.00(193.00,296.50)$ & 0.02 \\
\hline RDW fluctuation & $17.44(5.46,31.20)$ & $1.16(0.63,3.48)$ & $<0.01$ \\
\hline
\end{tabular}

GA: gestation age; BW: birth weight; WBC: White Blood Cells; NLR: Neutrophil to Lymphocyte ratio; RDW_initial: The first Red Cell Distribution Width after admission to NICU; RDW_max: The maximum of RDW detected in NICU; RDW_min: The minimal of RDW detected in NICU; RDW fluctuation: (RDW_max-RDW_initial)/RDW_initial *100; PLT: Platelet; RPR: RDW_initial to platelet ratio; RDS: Respiratory Distress Syndrome PDA: Patent Ductus Arteriosus LOS: Length of stay in NICU 


\begin{tabular}{|c|c|c|c|}
\hline & Sepsis group & Control group & $\mathbf{P}$ \\
\hline & $N=127$ & $N=127$ & \\
\hline $\mathrm{RPR}(\mathrm{IQR})$ & $0.08(0.06,0.10)$ & $0.07(0.05,0.09)$ & 0.03 \\
\hline Comorbidity & & & \\
\hline RDS, n (\%) & $113(89.00)$ & $69(54.30)$ & $<0.01$ \\
\hline PDA, n (\%) & $55(43.30)$ & $20(15.70)$ & $<0.01$ \\
\hline LOS, d(IQR) & $65.12(34.56,95.75)$ & $17.71(6.38,41.28)$ & $<0.01$ \\
\hline In-hospital mortality (\%) & $5(3.90)$ & $6(4.70)$ & 1.00 \\
\hline $\begin{array}{l}\text { GA: gestation age; BW: b } \\
\text { RDW_initial: The first Rec } \\
\text { of RDW detected in NICU } \\
\text { (RDW_max-RDW_initial)/ } \\
\text { Respiratory Distress Syn }\end{array}$ & $\begin{array}{l}\text { ght; WBC: White Blo } \\
\text { stribution Width af } \\
\text { min: The minimal o } \\
\text { hitial *100; PLT: Pla } \\
\text { DA: Patent Ductus }\end{array}$ & $\begin{array}{l}\text { R: Neutrophil to Ly! } \\
\text { n to NICU; RDW_me } \\
\text { cted in NICU; RDW f } \\
\text { RDW_initial to platel } \\
\text { LOS: Length of stay }\end{array}$ & $\begin{array}{l}\text { ratio; } \\
\text { ximum } \\
\text { S: }\end{array}$ \\
\hline
\end{tabular}

The sepsis group in the case-control matching had significantly higher maximum RDW [20.20\% (18.30, $22.00)$ vs. $17.40 \%(16.50,18.75)]$, RDW fluctuation $[17.44 \%(5.46,31.20)$ vs. $1.16 \%(0.63,3.48)]$, and RPR levels $[0.08(0.06,0.10)$ vs. $0.07(0.05,0.09)](P<0.05)$. The incidence of RDS was higher in the sepsis group $(89.00 \%$ vs. $54.30 \%, P<0.01)$ than the control group. Similarly, the incidence of PDA was higher in the sepsis group $(43.30 \%$ vs. $15.70 \% ; P<0.01)$. The length of stay in the NICU was significantly longer in the sepsis group $(P<0.01)$.

\section{Accuracy of RDW for diagnosing sepsis}

Figure $1 \mathrm{C}$ and Table 3 show the ROC curve of RDW and RPR for sepsis. RDW fluctuation showed better sensitivity and specificity than maximum RDW (AUC: 0.88 vs. 0.81 , sensitivity: $92.10 \%$ vs. $78.70 \%$; specificity: $71.70 \%$ vs. $69.30 \%$, respectively). The AUC of RPR was not statistically significant $(0.57, P=$ 0.05). (Table 3) 
Table 3

The performance of RDW_max, RDW fluctuation, and RPR in sepsis diagnosis

\begin{tabular}{|c|c|c|c|c|c|c|}
\hline Parameters & $P$ & AUC & $95 \% \mathrm{Cl}$ & Cut-off value (\%) & $\begin{array}{l}\text { Sensitivity } \\
\text { (\%) }\end{array}$ & $\begin{array}{l}\text { Specificity } \\
\text { (\% ) }\end{array}$ \\
\hline RDW fluctuation & 0.000 & 0.88 & $0.84-0.92$ & 1.94 & 92.10 & 71.70 \\
\hline RDW_max & 0.000 & 0.81 & $0.76-0.87$ & 18.15 & 78.70 & 69.30 \\
\hline RPR & 0.05 & 0.57 & $0.50-0.64$ & ND & ND & ND \\
\hline
\end{tabular}

Table 4

Univariate and multivariate logistic regression analysis for RDW predicting sepsis in case-control data

\begin{tabular}{|lll|}
\hline Variable & OR $95 \% \mathrm{Cl}$ & P \\
\hline RDW fluctuation & $1.17(1.12-1.22)$ & $<0.01$ \\
\hline RDW_max & $1.85(1.57-2.18)$ & $<0.01$ \\
\hline RDW fluctuation & & $<0.01$ \\
\hline RDW_max & $1.13(1.08-1.18)$ & $<0.01$ \\
\hline $\begin{array}{l}\text { a: adjusted for birth weight, White Blood Cells, Hemoglobin, Platelet; RDW_max: The maximum of } \\
\text { RDW detected in NICU; RDW fluctuation: (RDW_max-RDW_initial)/RDW_initial *100 }\end{array}$ & \\
\hline
\end{tabular}

\section{Univariate and Multivariate Analysis.}

Univariate and multivariate logistic regression showed that the maximum RDW and RDW fluctuation were independently associated with sepsis [odds ratio (OR) $1.61,95 \% \mathrm{Cl} 1.34-1.92, P<0.01 ; 0 \mathrm{O} 1.13,95 \% \mathrm{Cl}$ $1.08-1.18, P<0.01$, respectively] (Table 4).

\section{Discussion}

Our results demonstrated that the maximum RDW and RDW fluctuation were associated with neonatal sepsis. RDW fluctuation showed better accuracy than maximum RDW, with a sensitivity of $92.10 \%$ and specificity of $71.70 \%$. Further, for every $1 \%$ relative increase in the maximum RDW during the NICU stay, the likelihood of sepsis rose by $61 \%$ (OR 1.61); for every $1 \%$ relative increase in RDW fluctuation, the likelihood of sepsis rose by $13 \%$ (OR 1.13).

Our results indicated that RDW values varied widely with GA. RDW values at 34-37 weeks in the control group were higher than in the other GAs. Similarly, a large-scale cohort study of 8089 individuals found a strong association between RDW and age, but not sex [10]. Another study of RDW values showed that 
RDW reference intervals for neonates are higher than older children and adults. The lower reference limit for RDW at birth for term and late preterm neonates is $15.5 \%$ and the upper reference limit is $20 \%$, slightly higher (up to $23 \%$ ) in preterm neonates. The study also found that the reference interval at birth does not change appreciably over the first two weeks, except for those receiving a blood transfusion where the RDW increases [11]. Moreover, another study showed that blood parameters of preterm neonates depend on both the degree of prematurity, postnatal age, and perinatal treatments [12]. An observational study of 1594 normal newborns whose samples were taken on the first day of life demonstrated that the normal range for RDW values at GA 32-34 weeks was higher than at 35-36 weeks and 37-42 weeks $(P=0.002$ and 0.003) [13]. Similarly, another study showed that RDW and GA at birth were negatively correlated [14]. Also, a study showed that the RDW values of newborns at $28 \pm 2$ gestational weeks with PDA and nonPDA were $(15.75 \pm 1.15)$ and $(16.85 \pm 1.49)$ respectively, with no significant difference [15]. A high RDW in neonates is likely due to reticulocytosis [11], possibly explaining the correlation between RDW and GA. In future studies, we will recruit a larger sample size to further explore the association between RDW and PDA and explain the greater change in RDW.

Similar to other studies [16], we found that RDW values varied by ethnicity. Mean and elevated RDW has progressively increased from 1999-2012 among adults in the United States, as observed among nonHispanic Whites, Blacks, and Mexican Americans [16]. Another study showed that the median RDW was distributed differently among various ethnicities [17]. This is another aspect to investigate in future studies.

Recognition of biomarkers with high sensitivity and specificity is important for the early diagnosis and for prognosticating neonatal sepsis. Several biomarkers such as procalcitonin, CRP, and interleukin 6 have been reported for the auxiliary diagnosis of neonatal sepsis, with a $72.3-90.8 \%$ sensitivity [18-20]. CRP is an acute-phase protein mostly produced by the liver following the onset of inflammation, but it rises after $12 \mathrm{~h}$ from the onset of sepsis and other non-infectious inflammatory diseases [21]. However, these biomarkers require extensive laboratory support systems and are difficult to perform when a limited volume of blood is available, as in the case of extremely preterm infants. RDW is a practical, simple, and accessible biomarker as a parameter of the routine complete blood count, which has a strong positive correlation with CRP [6].

Here, we found that the RDW value in the sepsis group was higher than that in the control group. Similarly, a prospective study measured RDW in 251 cases of neonatal sepsis that showed significantly higher RDW (19.9\% vs. $18.9 \%, P<0.001)$ than age-matched healthy controls [8]. Additionally, the diagnostic value of RDW was studied recently for early-onset neonatal sepsis cases, and it was found that the mean RDW values was higher $(19.2 \pm 2.9)$ in the sepsis group than in the control group (16.9 \pm $1.3, P<0.01)[22]$. These findings suggest that elevated RDW was associated with neonatal sepsis.

Our study found that an RDW cut-off of $18.15 \%$ was associated with a sensitivity of $78.70 \%$ and a specificity of $69.30 \%$ for predicting neonatal sepsis $(P<0.01)$. Previous studies have shown that RDW has high diagnostic accuracy for sepsis [23]. A cut-off value of $>19.50 \%$ had a sensitivity of $87 \%$ and a 
specificity of $81 \%$ for late-onset Gram-negative sepsis $(P<0.001)$. They enrolled 47 infants with Grampositive sepsis and 39 infants with Gram-negative sepsis, whose median GA was 28 (range 26-31) weeks. In our study, the median GA was 33.50 (range 30-34.5) weeks. They found that RDW levels remained unchanged in infants with Gram-positive sepsis $(P=0.4)$. We did not compare RDW values between infants with Gram-positive and Gram-negative infections. Future studies should investigate the effect of different pathogens on RDW. High RDW may indicate high-risk newborns [14].

In our study, RDW fluctuation was found to be more useful for diagnosing neonatal sepsis than the maximum RDW. The sensitivity of the RDW fluctuation with a cut-off value of 1.94 was $92.10 \%$, and its specificity was $71.70 \%$. For long-term hospitalized premature infants, RDW fluctuations were more readily obtainable than blood culture results without collecting additional blood for testing. Using RDW allows early identification of sepsis for prompt treatment, thus improving the prognosis and decreasing patient mortality. Furthermore, multivariable logistic regression analysis of RDW fluctuation, birth weight, WBC, hemoglobin, and platelet count showed a positive independent association between RDW fluctuation and sepsis diagnosis (OR, 1.13; $95 \% \mathrm{Cl}, 1.08-1.18)$. Kim et al. (2013) found that, in adults, an increase in RDW from baseline during the first 72 hours of hospitalization is significantly associated with adverse clinical outcomes and suggested that a combination of baseline RDW value and an increase in RDW can be a potential independent prognostic marker in patients with severe sepsis or septic shock [24]. Nevertheless, the pathogenesis between RDW and outcomes of RDW is still unclear. Inflammation and organ dysfunction are two possible mechanisms [25]. Inflammation impairs RBC maturation, accelerating the entry of immature RBCs into the circulation [26]. RDW is determined by the life span of RBC [27]. The half life span of RBC is about 120 days, thus RDW may serve as a long-term inflammatory marker. In contrast, traditional markers such as CRP are only short-term markers. For patients with sepsis, higher RDW would indicate probable organ dysfunction, which may have worse outcomes.

To the best of our knowledge, this was the first study in which the relationship between RDW fluctuation and diagnosis of neonatal sepsis has been evaluated using data from the MIMIC- $\llbracket$ database. Our study, however, still has some limitations. First, weaknesses of our study are inherent to its retrospective nature. The MIMIC III database has some incomplete data, thus, analysis for confounding factors is incomplete. Second, we used the search terms "bacteremia of newborn" and "septicemia [sepsis] of newborn" for sepsis; therefore, the blood culture results were unclear. Finally, other risk factors such as erythrocyte transfusions [12], were not included in our study; thus, residual confounding factors remain possible. To increase the credibility of our results, we compared the RDW of patients with sepsis with those of matched controls based on GA, sex, and ethnicity. Future studies should include a larger sample size and prospective design to validate the findings of the present study.

\section{Conclusions}

In conclusion, RDW fluctuations in neonates with sepsis may be an independent outcome predictor for neonatal sepsis. This allows early identification and management of septic neonates. We suggest that RDW measurement should be considered as a diagnostic marker for the assessment of NICU patients 
with sepsis. Abbreviations: IRB: Institutional Review Board; IQR: The median interquartile range; LOS: length of stay in NICU; MIMIC-III: Medical Information Mart for Intensive Care III; NICU: neonatal ICU; OR: odds ratio; PDA: Patent Ductus Arteriosus; RDW: red cell distribution width RBC: red blood cell; RPR: RDW

to platelet ratio; RDS: respiratory distress syndrome; ROC: The receiver operating characteristic curve; WBC: white blood cell.

\section{Declarations}

Ethics approval and consent to participate: Not applicable

Consent for publication: Not applicable Availability of data and materials: The datasets used and/or analyzed during the current study are available from the corresponding author on reasonable request.

Competing interests: The authors declare that they have no competing interests.

Funding: Not applicable

Authors' contributions: Bin-Fang Guo designed the investigation, extracted data, analysed data and drafted the manuscript. Yu-Chun Wang and Ping-Ping Xu revised the manuscript and approved the submission. The author(s) read and approved the final manuscript.

Acknowledgements: Not applicable

Authors' information: Department of Neonatology, Cangzhou Central Hospital, No. 16, Xinhua Road, Yunhe District, Cangzhou, Hebei 061000, China

\section{References}

1. Cohen J, Vincent JL, Adhikari NKJ, Machado FR, Angus DC, Calandra T, et al. Sepsis: a roadmap for future research. Lancet Infect Dis. 2015;15:581-614.

2. Lippi G, Plebani M. Red blood cell distribution width (RDW) and human pathology. One size fits all. Clin Chem Lab Med. 2014;52:1247-9.

3. Salvagno GL, Sanchis-Gomar F, Picanza A, Lippi G. Red blood cell distribution width: a simple parameter with multiple clinical applications. Crit Rev Clin Lab Sci. 2015;52:86-105.

4. Vayá A, Sarnago A, Fuster O, Alis R, Romagnoli M. Influence of inflammatory and lipidic parameters on red blood cell distribution width in a healthy population. Clin Hemorheol Microcirc. 2015;59:37985.

5. Lippi G, Tagher G, Montagnana M, Salvagno GL, Zoppini G, Guidi GC. Relation between red blood cell distribution width and inflammatory biomarkers in a large cohort of unselected outpatients. Arch of Pathol Lab Med. 2009;133:628-32.

6. Ellahony DM, El-Mekkawy MS, Farag MM. A study of red cell distribution width in neonatal sepsis. Pediatr Emerg Care. 2017;36:378-88. 
7. Zhang HB, Chen J, Lan QF, Ma XJ, Zhang SY. Diagnostic values of red cell distribution width, platelet distribution width and neutrophil-lymphocyte count ratio for sepsis. Exp Ther Med. 2016;12:2215-9.

8. Martin SL, Desai S, Nanavati R, Colah RB, Ghosh K, Mukherjee MB. Red cell distribution width and its association with mortality in neonatal sepsis. J Matern Fetal Neonatal Med. 2019;32:1925-30.

9. Johnson AE, Pollard TJ, Shen L, Lehman LH, Feng M, Ghassemi M, et al. MIMIC-III, a freely accessible critical care database. Sci Data. 2016;3:1-9.

10. Hoffmann JJML, Nabbe KCAM, van den Broek NMA. Effect of age and gender on reference intervals of red. blood cell distribution width (RDW) and mean red cell volume (MCV). Clinical Chemistry and Laboratory Medicine (CCLM). 2015; 53:2015-9.

11. Christensen RD, Yaish HM, Henry E, Bennett ST. Red blood cell distribution width: reference intervals for neonates. J Matern Fetal Neonatal Med. 2015;28:883-8.

12. Roudil P, Vasselon C, Trombert-Paviot B, Berger C, Patural H. Blood parameters of preterm neonates: postnatal evolution according to gestational age. Int J Lab Hematol. 2017;39:317-28.

13. Tonbul A, Tayman C, Catal F, Kara S, Tatil MM. Red cell distribution width (RDW) in the newborn: normative data. J Clin Lab Anal. 2011;25:422-5.

14. Garofoli F, Ciardelli L, Mazzucchelli I, Borghesi A, Angelini M, Bollani L, et al. The red cell distribution width (RDW): Value and role in preterm, IUGR (intrauterine growth restricted), full-term infants. Hematology. 2014;19:365-9.

15. Özer Bekmez B, Tayman C, Büyüktiryaki M, Çetinkaya AK, Çakır U, Derme T. A promising, novel index in the diagnosis and follow-up of patent ductus arteriosus: red cell distribution width-to-platelet ratio. J Clin Lab Anal. 2018;32:e22616.

16. Loprinzi PD, Loenneke JP, Ahmed HM, Blaha MJ. Sex and race-ethnicity secular trends in mean and elevated red blood cell distribution width among adults in the United States, 1999-2012. Ethn Dis. 2016;26:45-50.

17. Kim J, Im JS, Choi CH, Park CH, Lee JI, Son KH, et al. The association between red blood cell distribution width and sarcopenia in U.S. adults. 2018; 8:11484.

18. Aydemir C, Aydemir H, Kokturk F, Kulah C, Mungan AG. The cut-off levels of procalcitonin and Creactive protein and the kinetics of mean platelet volume in preterm neonates with sepsis. BMC Pediatr. 2018;18:253.

19. Eschborn S, Weitkamp JH. Procalcitonin versus C-reactive protein: review of kinetics and performance for diagnosis of neonatal sepsis. J Perinatol. 2019;39:893-903.

20. Frerot A, Baud O, Colella M, Taibi L, Bonacorsi S, Alberti C, et al. Cord blood procalcitonin level and early-onset sepsis in extremely preterm infants. Eur J Clin Microbiol Infect Dis. 2019;38:1651-7.

21. Laborada G, Rego M, Jain A, Guliano M, Stavola J, Ballabh P, et al. Diagnostic value of cytokines and C-reactive protein in the first 24 hours of neonatal sepsis. Am J Perinatol. 2003;20:491-501.

22. Karabulut B, Arcagok BC. New diagnostic possibilities for early onset neonatal sepsis: red cell distribution width to platelet ratio. Fetal Pediatr Pathol. 2020;39:297-306. 
23. Park SH, Park CJ, Lee BR, Nam KS, Kim MJ, Han MY, et al. Sepsis affects most routine and cell population data (CPD) obtained using the Sysmex XN-2000 blood cell analyzer: neutrophil-related CPD NE-SFL and NE-WY provide useful information for detecting sepsis. Int J Lab Hematol. 2015;37:190-8.

24. Kim CH, Park JT, Kim EJ, Han JH, Choi JY, Han SH, et al. An increase in red blood cell distribution width from baseline predicts mortality in patients with severe sepsis or septic shock. Crit Care. 2013;17:R282.

25. Hu ZD, Lippi G, Montagnana M. Diagnostic and prognostic value of red blood cell distribution width in sepsis: a narrative review. Clin Biochem. 2020;77:1-6.

26. Montagnana M, Cervellin G, Meschi T, Lippi G. The role of red blood cell distribution width in cardiovascular and thrombotic disorders. Clin Chem Lab Med. 2012;50:635-41.

27. Shemin D, Rittenberg D. The life span of the human red blood cell. J Biol Chem. 1946;166:627-36.

\section{Figures}




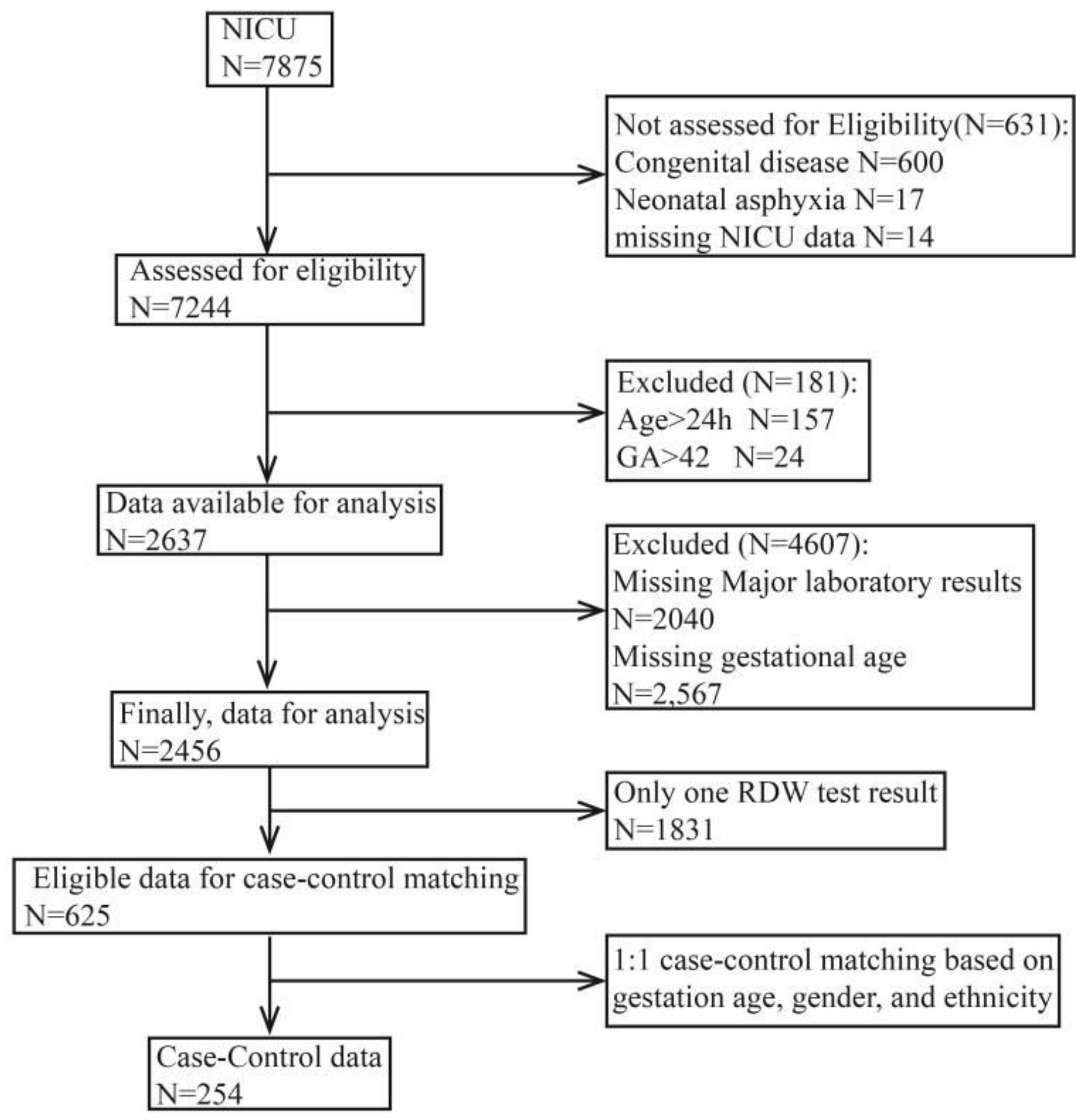

Figure 1

Flowchart 


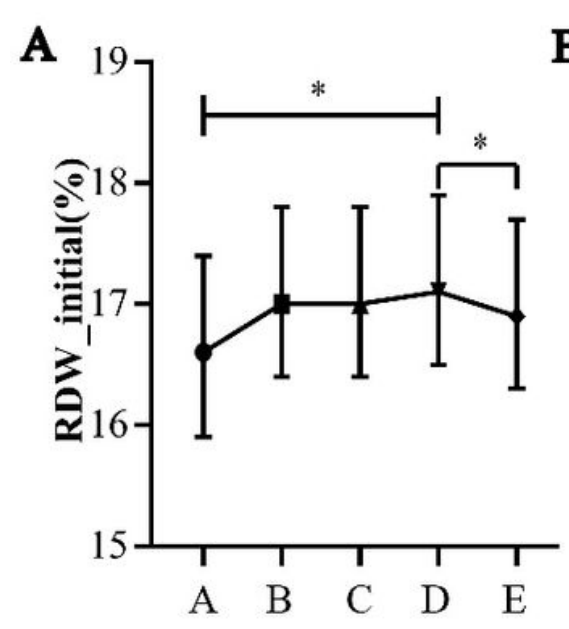

Gestational Age

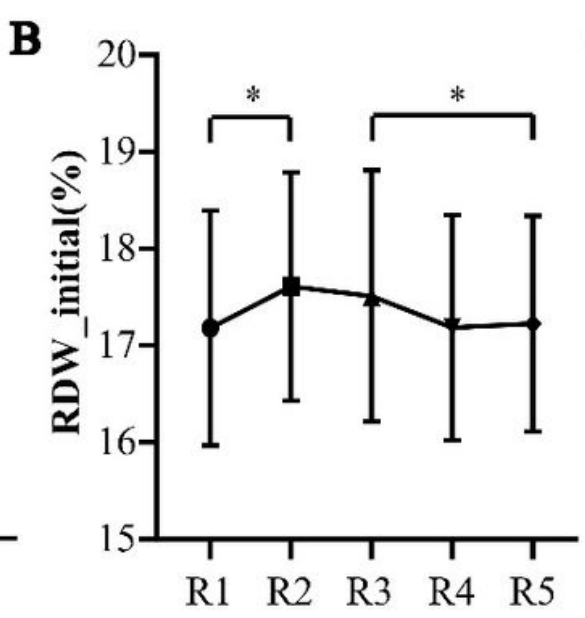

Ethnicity

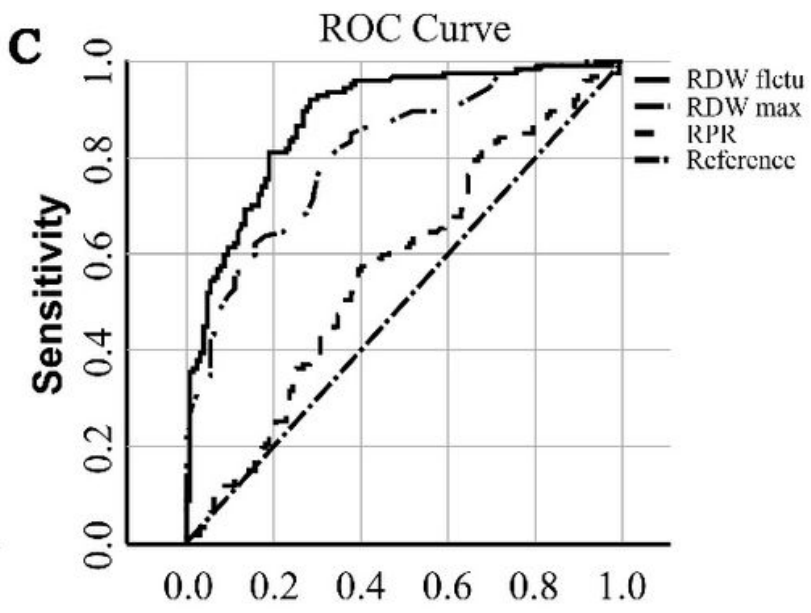

Specificity

Figure 2

Effects of gestational age (GA), ethnicity on the initial of RDW and Receiver operating characteristics Curve of RDW for predicting sepsis A: the minimal of RDW between different gestational ages (GA) in the control group A: GA<28; B: 32>GA 228 ; C: 34>GA $\geq 32 ; D: 37>G A \geq 34 ; E: G A \geq 37$ B: the minimal of RDW between different ethnicity in the control group with gestational age between 34 and 37 weeks R1: other or unknown; R2: Hispanic; R3: Black; R4: Asian; R5: Caucasian The middle line represents the median, and the bars represent the interquartile ranges (from the 75th percentile to the 25th percentile) $\Downarrow$ : $P<0.05 \mathrm{C}$ : receiver operating characteristics Curve of RDW_max, RDW fluctuation, and RPR for predicting sepsis RDW_initial: The first Red Cell Distribution Width after admission to NICU; RDW fluctuation: (RDW_maxRDW_initial)/RDW_initial *100; RDW_max: The maximum of RDW detected in NICU; RPR: RDW_initial to platelet ratio 